\title{
The psoriatic shift induced by interleukin 17 is promptly reverted by a specific anti-IL-17A agent in a three-dimensional organotypic model of normal human skin culture
}

\author{
Elena Donetti, ${ }_{1}^{1}$ Giulia Lombardo, ${ }^{1}$ Serena Indino, ${ }^{1}$ Laura Cornaghi, ${ }^{1}$ Francesca Arnaboldi, ${ }^{1}$ Leonardo Pescitelli, ${ }^{2}$ \\ Franz Baruffaldi Preis, ${ }^{3}$ Francesca Prignano ${ }^{2}$
}

${ }^{1}$ Department of Biomedical Sciences for Health, University of Milan

${ }^{2}$ Department of Health Sciences, University of Florence

${ }^{3}$ Plastic Surgery Unit, Ospedale Niguarda Ca' Granda, Milan, Italy

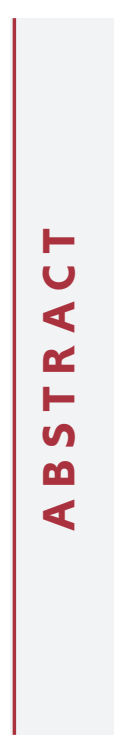

\begin{abstract}
Interleukin 17A (IL-17A), mainly produced by the T helper subclass Th17, plays a key role in the psoriatic plaque formation and progression. The clinical effectiveness of anti-IL-17A agents is documented, but the early and specific mechanisms of their protection are not identified yet. The challenge of the present study is to investigate the possible reversal exerted by a specific anti-IL-17A agent on the psoriatic events induced by IL-17A in a three-dimensional organotypic model of normal human skin. Bioptic skin fragments obtained after aesthetic surgery of healthy women ( $n=5)$ were incubated with i) IL-17A biological inhibitor (anti-IL-17A), ii) IL$17 \mathrm{~A}$, iii) a combination of IL-17A and its specific IL-17A biological inhibitor (COMBO). A Control group was in parallel cultured and incubation lasted for 24 and $48 \mathrm{~h}$ epidermal-side-up at the air-liquid interface. All subjects were represented in all experimental groups at all considered time-points. Keratinocyte proliferation and the presence of epidermal Langerhans cells were quantitatively estimated. In parallel with transmission electron microscopy analysis, immunofluorescence studies for the epidermal distribution of keratin (K)10, K14, K16, K17, filaggrin/occludin, Toll-like Receptor 4, and Nuclear Factor kB were performed. IL-17A inhibited cell proliferation and induced K17 expression, while samples incubated with the anti-IL-17A agent were comparable to controls. In the COMBO group the IL-17A-induced effects were almost completely reverted. Our study, for the first time, elucidates the most specific psoriatic cellular events that can be partially affected or completely reverted by a specific anti-IL-17A agent during the early phases of the plaque onset and progression. On the whole, this work contributes to expand the knowledge of the psoriatic tableau.
\end{abstract}

Key words: Langerhans cells; keratinocytes, keratin 17; cell proliferation; transmission electron microscopy; epidermal barrier.

Correspondence: Prof. Elena Donetti, Department of Biomedical Sciences for Health, University of Milan, Via Mangiagalli 31, 20133 Milan, Italy. Tel. +39.02.50315400 - Fax: +39.02.50315387.

E-mail: elena.donetti@unimi.it

Contributions: ED, FP, designed the experimental study and wrote the manuscript; ED, carried out the transmission electron microscopy analysis; GL, SI, performed all the immunofluorescence experiments; LC, performed the quantitative analysis of epidermal proliferation; FA, performed the quantitative analysis of epidermal Langerhans cells; LP, FBP, significantly contributed to the discussion. All the authors have read and approved the final version of the manuscript and agreed to be accountable for all aspects of the work.

Conflict of interest: The Authors declare no conflict of interest.

Funding: This research did not receive any specific grant from funding agencies in the public, commercial, or not-for-profit sectors.

Availability of data and materials: The datasets used and/or analyzed during the current study are available from the corresponding author on reasonable request.

Ethical Approval: All procedures performed in studies involving human participants were in accordance with the ethical standards of the Institutional Research Committee and with the 1964 Helsinki declaration and its latest amendment.

Informed consent: Written informed consent was obtained all patients. 


\section{Introduction}

A complex and fascinating interplay among circulating cytokines and different epidermal cytotypes leads to the formation and to the progression of the psoriatic plaque. Most of the literature has produced great information concerning $\mathrm{T}$ cell maturation and modifications induced by interleukin-17 (IL-17), especially regarding the specific pathogenic role of IL-17A isoform in psoriasis. ${ }^{1}$ Much fewer data take into account the early epidermal injury of IL-17A on the physical/immune epidermal barrier and the terminal differentiation, as well as the possible recovery after administration of anti-IL-17A.2,3 Although the clinical effectiveness of anti-IL-17A agents is widely reported, the cellular mechanisms underlying their protection are not identified yet. A good possibility to complete this tableau springs from the three-dimensional (3D) organotypic model of normal human skin culture. This experimental approach is a simple and very clean setting allowing the study of the sharp epidermal changes induced by a stimulus, in this case IL-17A, with any involvement of neither blood nor lymphatic supply. Keratinocytes (KCs) and Langerhans cells (LCs) are the two main epidermal cytotypes implicated in organizing a fast and specific response to overcome the possible insult. ${ }^{4,5}$ Epidermal $\mathrm{KCs}$ are characterized by an abundant cytoplasmic network of keratin $(\mathrm{K})$ intermediate filaments, ranging from $10 \%$ of total proteins in the basal proliferative layer to $70 \%$ in the uppermost epidermal layers, thus reflecting a dynamic regulation through the differentiation process. $^{6}$ During this process, K5/K14 are typically expressed in the proliferative basal compartment, while $\mathrm{K} 1 / \mathrm{K} 10$ in the suprabasal differentiating layers. Keratins have been recognized as regulators of many cell functions and as mediators of the epidermal integrity maintenance after a stress. ${ }^{7}$ A mechanical skin injury significantly affects keratin expression. ${ }^{8}$ In particular, K16 and K17 were indicated as "psoriatic keratins" in the differentiated epidermal layers as they are associated with epidermal hyperproliferation. ${ }^{9}$ Simultaneously, further processes possibly occur within the "injured" epidermis to promote a defense response: epidermal LCs can be recruited, accumulated, and finally activated for the later migration. In parallel, a specific expression of Toll-like Receptors (TLRs) can follow, thus enhancing the binding with specific ligands, with the consequent activation of the transcription factor Nuclear Factor kB (NFkB) and translocation from the cytoplasm to the nucleus. ${ }^{10}$

The challenge of the present study is to investigate the effects of an IgG1 monoclonal antibody anti-IL-17A (anti-IL-17A agent) on the morphological psoriatic events, directly induced by IL-17A, in the 3D organotypic model of normal human skin culture, previously standardized in our lab..$^{4,5,11-13}$ Specific attention was payed to keratin expression and to the integrity of the physical/immune epidermal barrier, as evaluated by immunofluorescence. Moreover, the ultrastructural analysis allowed evaluating morphological features of activated LCs and, again by immunofluorescence, TLR4 expression was taken into account.

\section{Materials and Methods}

\section{D organotypic model of normal human skin culture}

Human skin samples were obtained from plastic aesthetic surgery of abdominal or mammary regions of healthy nonsmoking 20 - to 40-year-old women $(\mathrm{n}=5)$; written informed consent was obtained from all subjects. Immediately after excision, the biopsies were reduced into 0.5 - to $1-\mathrm{cm}^{2}$ fragments using a sterile scalpel. All fragments were placed epidermal-side-up at the air-liquid inter- face in a Transwell system (Costar, Corning, NY, USA) and maintained at $37^{\circ} \mathrm{C}$ and $5 \% \mathrm{CO}_{2}$ overnight, in order to reduce the acute effects of surgery. Samples were cultured in Dulbecco's modified Eagle's culture medium (Euroclone, Milan, Italy) containing 10\% fetal bovine serum (Invitrogen, Life Technologies Ltd., Paisley, UK) supplemented with penicillin/streptomycin, amphotericin B and glutamine (Sigma-Aldrich, St. Louis, MO, USA). No hydrocortisone was added to avoid an anti-inflammatory activity on epidermal KCs as previously described. ${ }^{4}$ In order to evaluate KC proliferation, $3 \mathrm{~h}$ prior to the end of the experiment the culture medium was enriched with $400 \mu \mathrm{mol} 5$-bromo-2'-deoxyuridine (BrdU), a nonradioactive analogue of thymidine selectively incorporated into the DNA of S-phase cells. Specimens were further divided in three different groups which were added $2 \mu \mathrm{g} / \mathrm{mL}$ of anti-IL-17A agent (anti-IL-17A group), $50 \mathrm{ng} / \mathrm{mL}$ IL-17A (IL-17A group) or a combination of both (COMBO group). Samples were harvested 24 (T24) and $48 \mathrm{~h}$ (T48) after cytokine addition and were processed in parallel for light and transmission electron microscopy. Control samples (CTRL) were cultured in normal medium. All bioptic samples were represented at each time point and in all experimental groups.

\section{Light microscopy analysis}

All specimens were fixed in 4\% paraformaldehyde buffered with $0.1 \mathrm{M}$ phosphate-buffered saline (PBS) $\mathrm{pH} 7.4$ for $5 \mathrm{~h}$ at room temperature (RT), dehydrated in an ascending scale of ethanols and paraffin embedded. Five-micrometer-thick serial sections were obtained using a rotary microtome (RM2245; Leica Microsystems $\mathrm{GmbH}$, Wetzlar, Germany). At least 40 slides/sample were obtained and five non-consecutive slides for each sample were stained with hematoxylin and eosin to observe the morphological features.

\section{Transmission electron microscopy analysis}

For transmission electron microscopy analysis, specimens $(2 \times 2 \mathrm{~mm})$ were immersion-fixed in $3 \%$ glutaraldehyde diluted in $0.1 \mathrm{M}$ Sorensen's phosphate buffer $(\mathrm{pH} 7.4)$ overnight at $4^{\circ} \mathrm{C}$. Each sample was then washed with Sorensen's phosphate buffer (3 washes of $30 \mathrm{~min}$ each), post-fixed in $1 \%$ osmium tetroxide in Sorensen's buffer, dehydrated through an ascending scale of acetone and embedded in Durcupan (Fluka, Milan, Italy). Ultrathin sections $(60 \mathrm{~nm})$ were obtained using an Ultracut ultramicrotome (Reichert Ultracut R; Leika, Wein, Austria) and stained with uranyl acetate/lead citrate before examination with a CM10 Philips electron microscope.

\section{Immunofluorescence staining}

In all experiments, paraffin sections were dewaxed, rehydrated in a descending scale of ethanols and treated for antigen unmasking and saturation of non-specific sites. Antigen retrieval was performed incubating slides in $\mathrm{Na}$ citrate buffer $\mathrm{pH} 6$ either in microwave at $750 \mathrm{~W}$ three times for $3 \mathrm{~min}$ (for K10, K16, K17 and TLR4) or in autoclave at $120^{\circ} \mathrm{C}$ for $6-10 \mathrm{~min}$ (for langerin, filaggrin, occludin and NFkB). BrdU detection implied DNA denaturation with $2 \mathrm{~N} \mathrm{HCl}$ (30 min), buffering with $\mathrm{Na}_{2} \mathrm{~B}_{4} \mathrm{O}_{7}(10 \mathrm{~min})$, and digestion with $0.05 \%$ pepsin in $0.02 \mathrm{M} \mathrm{HCl}(25 \mathrm{~min})$. The following primary antibodies were used for detection and incubated overnight at $4^{\circ} \mathrm{C}$ with the exception of monoclonal anti-BrdU (Santa Cruz Biotechnology, Santa Cruz, CA, USA; dilution 1:200; $1 \mathrm{~h}$ at RT), mouse anti-human keratin 16 (BioSB, dilution 1:100; $1 \mathrm{~h}$ at $37^{\circ} \mathrm{C}$ ), and mouse anti-human TLR4 (Novus Biologicals, Centennial, CO, USA; dilution $1: 300 ; 1 \mathrm{~h}$ at $37^{\circ} \mathrm{C}$ ): rabbit antihuman filaggrin (Sigma-Aldrich; dilution 1:2500); mouse antihuman keratin 10 (Novus Bio; dilution 1:50); mouse anti-human keratin 14 (Santa Cruz; dilution 1:100); rabbit anti-human keratin 17 (Sigma-Aldrich; dilution 1:500); rabbit anti-human occludin 
(Sigma-Aldrich; dilution 1:100); mouse anti-human langerin (Santa Cruz; dilution 1:50); rabbit anti-human NFkB (Santa Cruz; dilution 1:50). The following secondary antibodies were used $1 \mathrm{~h}$ at RT to develop immunofluorescence: Alexa Fluor 488 goat antirabbit $\operatorname{IgG}(\mathrm{H}+\mathrm{L})$ antibody (dilution 1:100) and goat anti-mouse FITC-conjugated (dilution 1:200) respectively for anti-K10, anti$\mathrm{K} 14$, anti-K16, anti-langerin and anti-TLR4 or for anti-filaggrin and anti-NFkB. One section on each slide was considered as technical negative control and the primary antibody was thus omitted and replaced with $0.1 \mathrm{M}$ PBS, pH 7.4. Nuclei were counterstained with 4',6-diamidino-2-phenylindole dihydrochloride (DAPI 1:50,000 dilution in bi-distilled water; $5 \mathrm{~min}$ of incubation at RT). After rinsing in PBS, sections were mounted with Mowiol 4-88 (Sigma-Aldrich). Immunofluorescence analysis were performed utilizing a Nikon Eclipse E600 equipped with a Nikon digital camera DXM1200 (Nikon, Tokyo, Japan). ${ }^{12}$ The analysis was aimed at locating the immunopositivity for the different proteins in the epidermal layers, without any attempts to estimate quantitatively the possible difference in the staining intensities.

\section{Quantitative analysis of keratinocyte proliferation}

For the quantitative analysis of $\mathrm{KC}$ proliferation, at least three immunofluorescence experiments were carried out for each sample, with two slides per sample and two sections on each slide (12 replicates for each sample). Two independent double-blinded

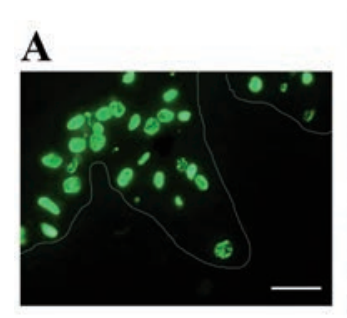

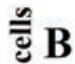

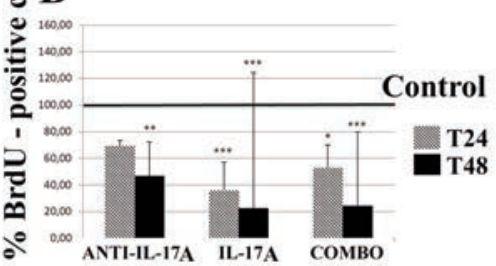

Treatment

Figure 1. Epidermal cell proliferation quantitative analysis. A) Representative BrdU immunofluorescence in normal human skin paraffin sections. B) Quantitative analysis of keratinocyte proliferation in skin samples treated with anti-IL-17A, IL-17A, or with COMBO. Results were expressed as percentage of BrdU-positive cells per $\mathrm{mm}^{2}$ of living epidermis +1 standard deviation considering untreated control samples as $\mathbf{1 0 0} \%$ (thick black line). Single asterisk indicates a statistically significant difference $(P<0.05)$ vs $T 24$ group. Double asterisk indicates a statistically significant difference $(\mathrm{P}<0.005)$ vs $\mathrm{T} 24$ group. Triple asterisk indicates a statistically significant difference $(\mathrm{P}<0.0001)$ vs $\mathrm{T} 24$ group. (One -way ANOVA test, Dunnett's post-test). Dotted white line in A indicates the basal membrane. T24: samples harvested after $24 \mathrm{~h}$ of culture; T48, samples harvested after $48 \mathrm{~h}$ of culture. Scale bar: $20 \mu \mathrm{m}$.

T24

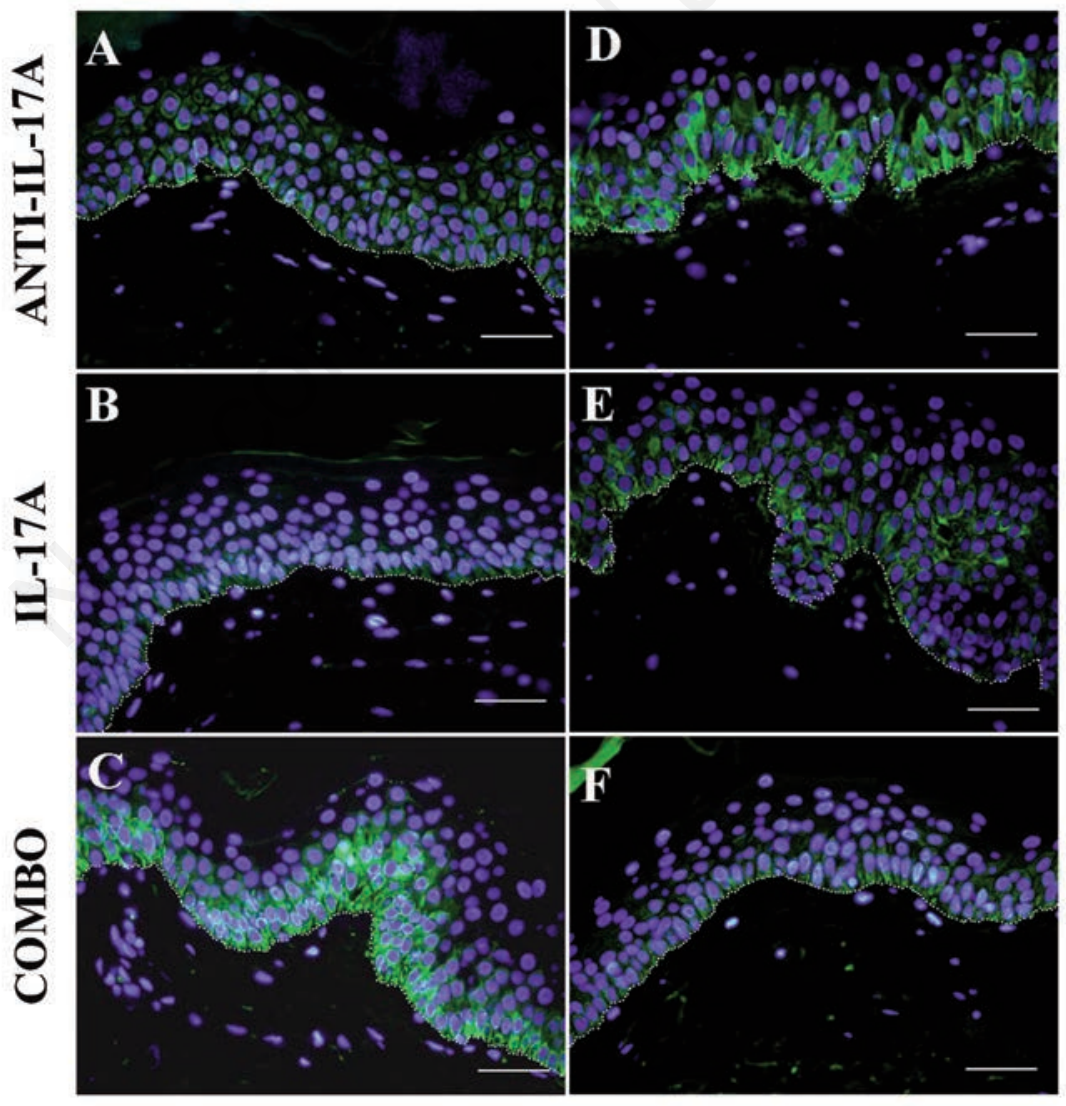

Figure 2. Keratin 16 immunofluorescence analysis. Representative photomicrographs of normal human skin paraffin sections after K16 immunofluorescence. A,D) IL-17A inhibitor-treated-samples harvested respectively after 24 (T24) and 48 (T48) h of culture. B,E) IL17A-treated-samples harvested respectively after 24 (T24) and 48 (T48) h of culture. C,F) COMBO samples harvested respectively after 24 (T24) and 48 (T48) h of culture. Nuclei are counterstained with DAPI. Dotted white line indicates the basal membrane. Scale bars: $50 \mu \mathrm{m}$. 
investigators counted the BrdU-positive cells. The epidermal area was calculated on adjacent hematoxylin and eosin-stained sections, excluding the stratum corneum. The software Image-Pro Plus (version 4.5.019; Media Cybernetics Inc., Silver Spring, MD, USA) was used for measurement following a previously standardized procedure. ${ }^{12}$ Results were expressed as the percentage of BrdU-positive cells per $\mathrm{mm}^{2}$ of living epidermis \pm 1 standard deviation, considering untreated control samples as $100 \%$. The statistically significant differences were obtained after carrying out the One-way ANOVA test, followed by Dunnett's post-test.

\section{Quantitative analysis of epidermal LCs}

For the quantitative analysis of LCs, at least 3 immunofluorescence experiments were carried out in all samples, with two slides per sample and two sections on each slide (12 replicates for each sample). Two independent double-blinded investigators counted the langerin-positive bodies of LCs. Epidermal area was calculated on adjacent hematoxylin and eosin-stained sections, excluding the stratum corneum, to normalize the immunofluorescence counts. For the area measurement the software Image-Pro Plus (version 4.5.019; Media Cybernetics Inc.) has been used following a previ- ously standardized procedure. ${ }^{10}$ Results were expressed as percentage of LCs $/ \mathrm{mm}^{2}$ of living epidermis +1 standard deviation considering untreated control samples as $100 \%$. The statistically significant differences were obtained after carrying out the one-way ANOVA test, followed by Dunnett's post-test.

\section{Results}

Immunoreactivity after the incubation with the anti-IL-17A agent was comparable to the observations already published for the control group concerning $\mathrm{K} 10$ and $\mathrm{K} 14,{ }^{4} \mathrm{~K} 17$ and occludin, ${ }^{5}$ langerin, filaggrin, and $\mathrm{NFkB},{ }^{13}$ respectively. Hence, these data referring to control group are not shown.

\section{Keratinocyte proliferation, K16 and K17 immunofluo- rescence}

BrdU immunostaining was always present as a punctuate staining in $\mathrm{KC}$ nuclei present in the basal layer (Figure 1A). Figure 1B reports the percentage inhibition of $\mathrm{KC}$ proliferation. In accor-

\section{T24}
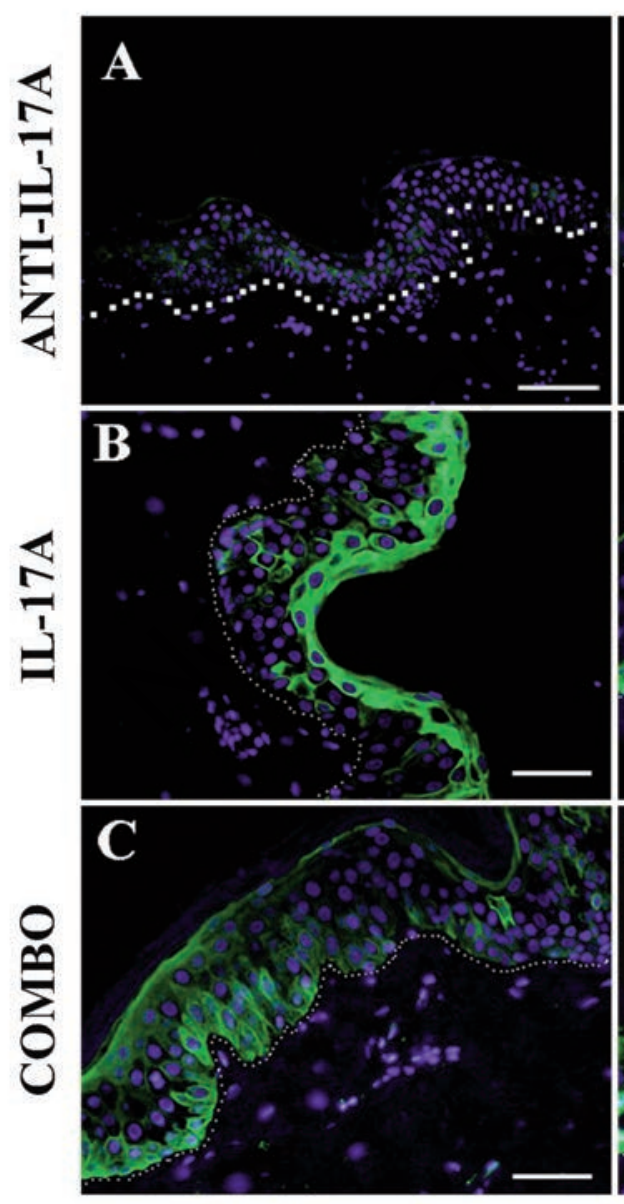

T48
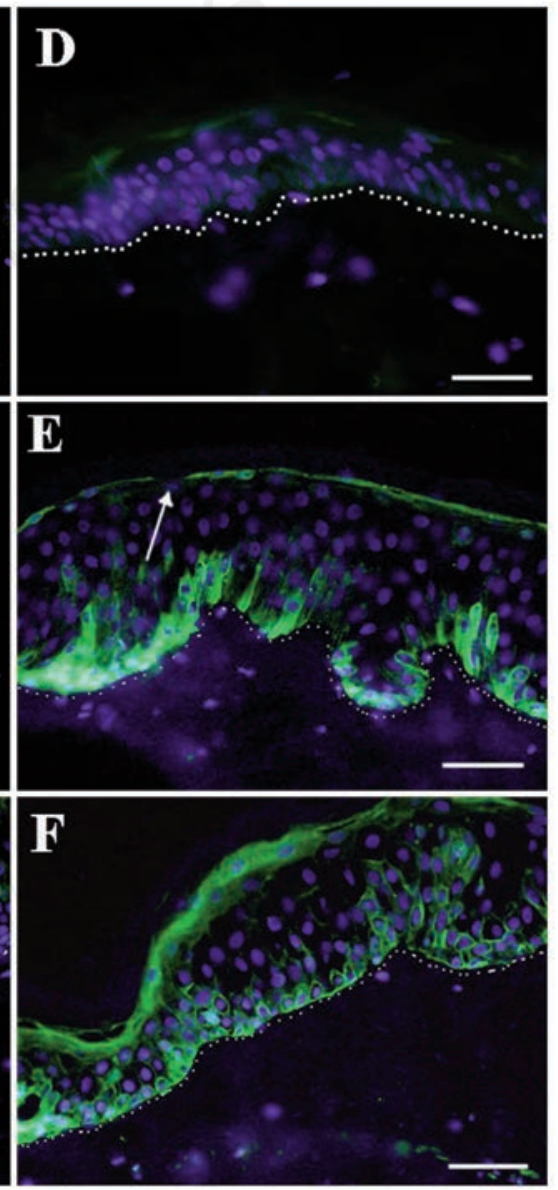

Figure 3. Keratin 17 immunofluorescence analysis. Representative photomicrographs of normal human skin paraffin sections after K17 immunofluorescence. A,D) IL-17A inhibitor-treated-samples harvested respectively after 24 (T24) and 48 (T48) h of culture. B,E) IL17A-treated-samples harvested respectively after 24 (T24) and 48 (T48) h of culture. C,F) COMBO harvested respectively after 24 (T24) and 48 (T48) h of culture. Nuclei are counterstained with DAPI. Dotted white line indicates the basal membrane. White arrow indicates the discontinuous immunostaining in the granular layer. Scale bars: $50 \mu \mathrm{m}$. 
dance with our previous results, ${ }^{4}$ IL-17A promptly inhibited cell proliferation at both time points. After the incubation with the antiIL-17A agent, an antiproliferative effect was evident starting from T24 and, even more, at T48. On the other hand, in COMBO group cell proliferation raised up only at $\mathrm{T} 24$, even though the proliferation rate levels observed in anti-IL-17A group were never restored. Although the variability was pronounced within each group, a statistically significant difference was always observed in all experimental groups at all considered time points.

K16 expression was completely absent in the more differentiated layers (Figure 2) after $24 \mathrm{~h}$ of incubation, with some dissimilarities regarding the basal and suprabasal compartments among the three experimental groups. In the IL-17A and the anti-IL-17A treated groups, a comparable pattern of K16 distribution resulted in the basal and in the lower spinous layers (Figure $2 \mathrm{~A}, \mathrm{~B}$ ). Unpredictably, in COMBO samples the immunostaining was clearly detectable both in basal and in suprabasal spinous layers (Figure 2C). At T48, the K16 distribution patterns found after the incubation with the anti-IL-17A agent alone or IL-17A alone were comparable with the expression found in the COMBO group at T24, i.e. in the basal and suprabasal epidermal layers. At T48 the COMBO group exhibited only a weak immunostaining in the basal layer (Figure 2F). If present, K17 immunostaining was always localized in the cytoplasm of the living epidermal compartments. In anti-IL-17A samples, K17 was never expressed in any epidermal layer (Figure 3 A,D). As expected, K17-positive epidermal cells were evident in IL-17A groups at both time points, with a different distribution pattern at T24 and T48 (Figure 3 B,E, respec- tively). At T24, a marked K17 immunostaining was detectable in the most differentiated KCs of the granular layer (Figure 3B). At $\mathrm{T} 48$, the basal layer resulted homogenously immunopositive for this marker (Figure 3E), while in the granular layer, the immunostaining was discontinuous (Figure 3E, white arrow). In the COMBO group, a weak K17 immunostaining was detectable in the basal and spinous layers at both time points (Figure $3 \mathrm{C}, \mathrm{F}$ ).

\section{Terminal differentiation}

In accordance with the literature data, K10 and K14 immunostainings were always localized in the cytoplasm of epidermal keratinocytes in the suprabasal and basal layers, respectively, without any difference among the experimental groups. The distribution of both K10 (Figure 4 A-F) and K14 (Figure 4 G-L) was unaffected by any treatment at all considered time-points.

\section{Physical epidermal barrier}

At T24, in anti-IL-17A group, filaggrin and occludin were continuously distributed in the $\mathrm{KC}$ cytoplasm of the granular layer (Figure 5 A,D). In IL-17A samples, occludin immunostaining was weakly detectable and discontinuous (Figure 5E, white arrows). In parallel, filaggrin immunostaining was less evident when compared with anti-IL-17A group (Figure 5B). In the COMBO group, filaggrin and occludin were homogeneously localized in the granular layer, showing a more intense and homogenoeus staining than in IL-17A group (Figure $5 \mathrm{C}, \mathrm{F}$ ) and a similar distribution in comparison to the anti-IL-17A group (Figure 5 A,D). Since at T48 in all experimental groups the overall distribution patterns of
T24
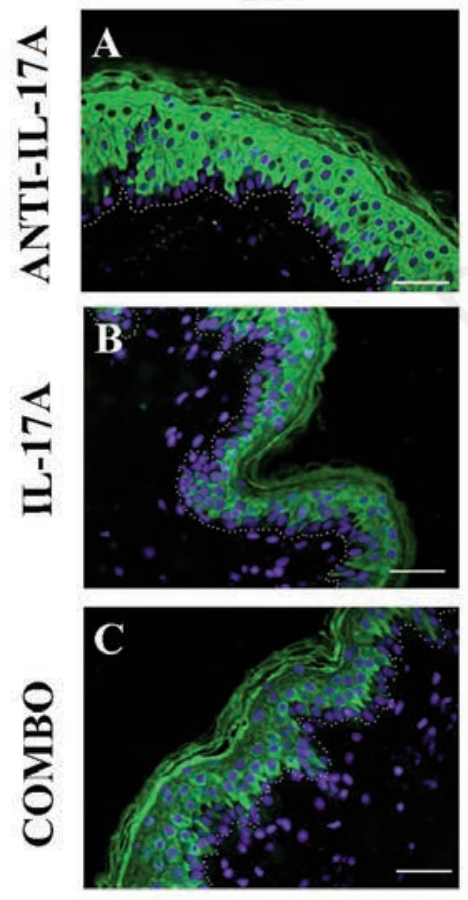

T48
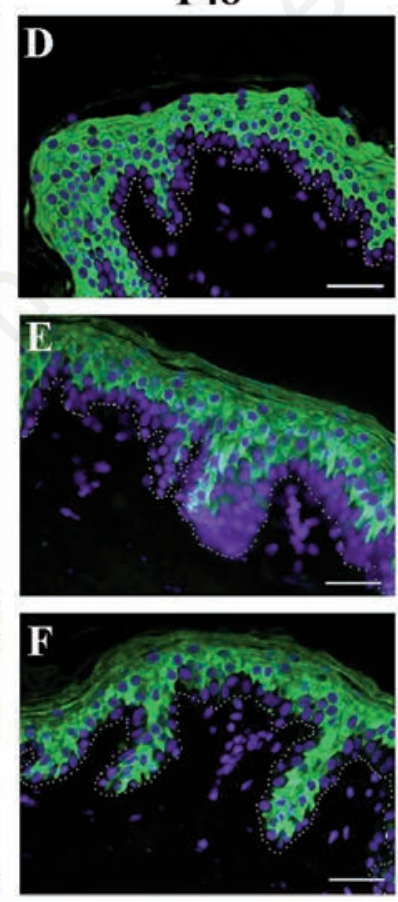

T24
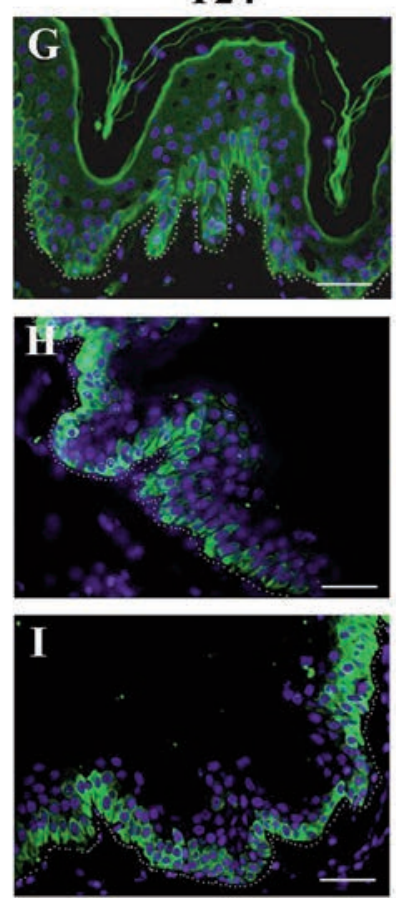

T48
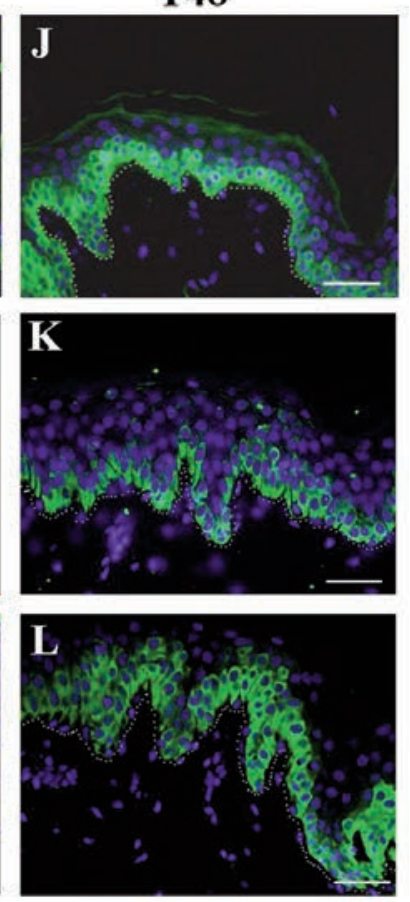

Figure 4. Keratin 10 and Keratin 14 immunofluorescence analysis. Representative photomicrographs of normal human skin paraffin sections after K10 (A-F) and K14 (G-L) immunofluorescence. A,G,D,J) IL-17A inhibitor-treated-samples harvested respectively after 24 (T24) and 48 (T48) h of culture. B,H,E,K) IL-17A -treated-samples harvested respectively after 24 (T24) and 48 (T48) h of culture. C,I,F,L) COMBO samples harvested respectively after 24 (T24) and 48 (T48) h of culture. Nuclei are counterstained with DAPI. Dotted white line indicates the basal membrane. Scale bars: $50 \mu \mathrm{m}$. 
occludin and filaggrin corresponded at T24 immunostaining, these data are not shown.

\section{Langerhans cells}

By immunofluorescence, we detected all LCs localized in the basal and suprabasal layers of epidermis and characterized by a stellate appearance, due to their dendritic processes originating from a large cellular body (Figure 6A). The quantitative analysis of epidermal LCs revealed a statistically significant decrease of LCs' number at both time points in anti-IL-17A group if compared to controls (fixed as $100 \%$, thick black line). In IL-17A samples, the number of LCs started to slightly decrease only after $48 \mathrm{~h}$, confirming our previously reported data. ${ }^{9}$ The incubation with both agents induced a not statistically different increase of the number of langerin-positive cells, if compared with IL-17A treated samples (Figure 6B).

The ultrastructural analysis helped to investigate LCs' activation (Figure $6 \mathrm{C}$-L). In the group exposed to the specific anti-IL17A agent, LCs' cytoplasm contained scattered Birbeck granules and there were no morphological signs of activation (Figure 6 C,D) and this tableau persisted up to $48 \mathrm{~h}$ (data not shown). When incubated with IL-17A, LCs always exhibited morphological cytoplasmic structures, as abundant Birbeck granules, indicating a clear activation (Figure 6 F,H, black arrows).

In the COMBO group, LCs appeared quiescent from a morphological point of view, without any evident sign of phenotype modification (Figure $6 \mathrm{~J}, \mathrm{~L}$ ). Along with LCs features, the enlargement of intercellular spaces induced by IL-17A incubation (Figure 6 E,G) was greatly reduced after the exposure to the specific antiIL-17A agent (Figure $6 \mathrm{I}, \mathrm{K}$ ). For completing the tableau of the innate immune epidermal barrier, TLR4 expression was evaluated by immunofluorescence. No differences were evident among all the experimental groups regarding TLR4 distribution pattern, which was confined to the cytoplasm of the epidermal basal layer (Figure 7).

Figure 8 reports the immunofluorescence analysis of the localization of the transcriptional factor NFkB. In all the experimental samples, NFkB staining was detectable in the KCs' cytoplasm.
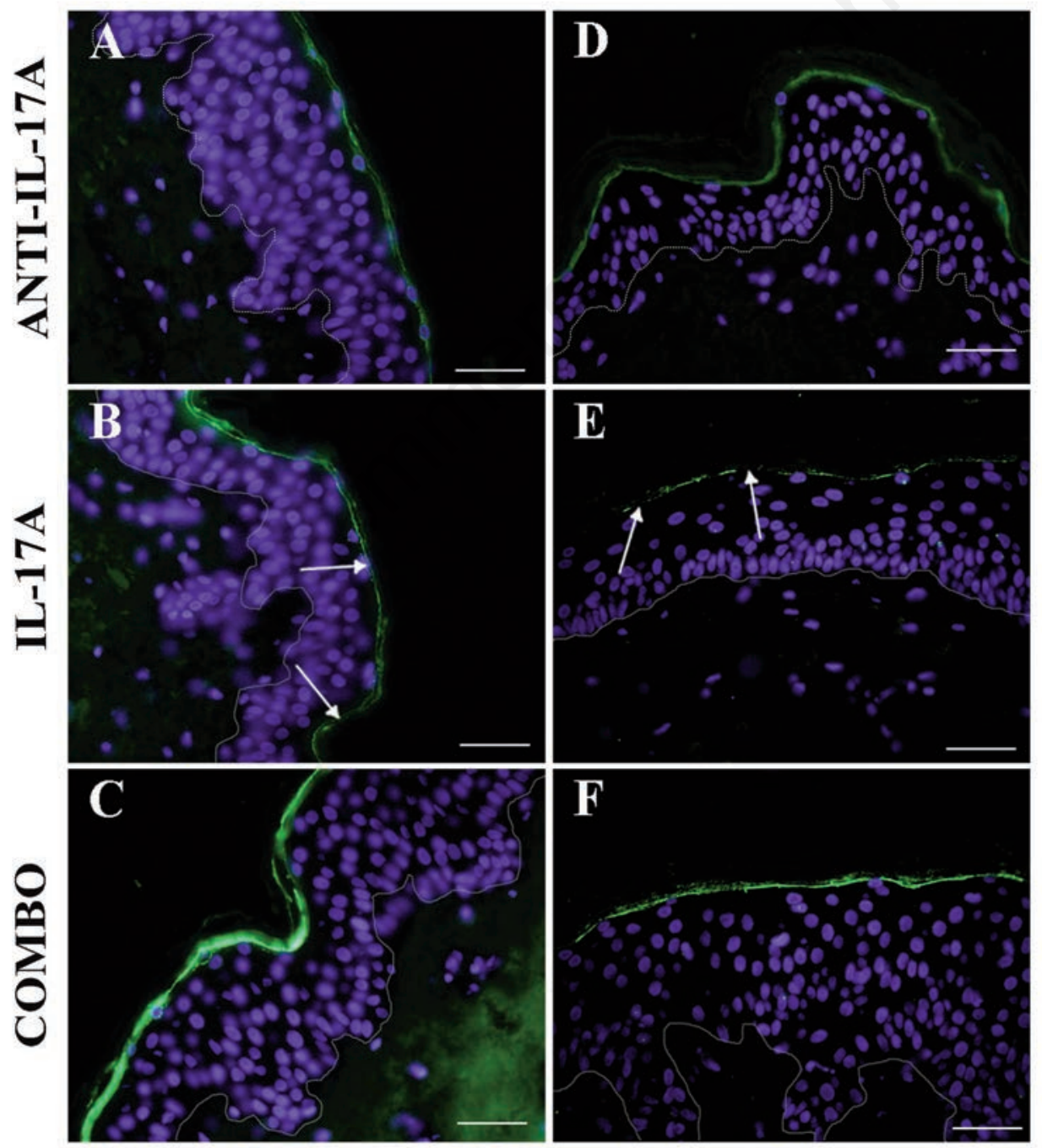

Figure 5. Filaggrin and occludin immunofluorescence analysis. Representative photomicrographs of normal human skin paraffin sections after filaggrin (A-C) and occludin (D-F) immunofluorescence in samples harvested after 24 h of culture (T24). A,D) IL-17A inhibitor-treated-samples. B,E) IL-17A -treated-samples. C,F) COMBO samples. Nuclei are counterstained with DAPI. Dotted white line indicates the basal membrane. White arrows indicate discontinuity of occludin immunofluorescence. Scale bars: $50 \mu \mathrm{m}$. 

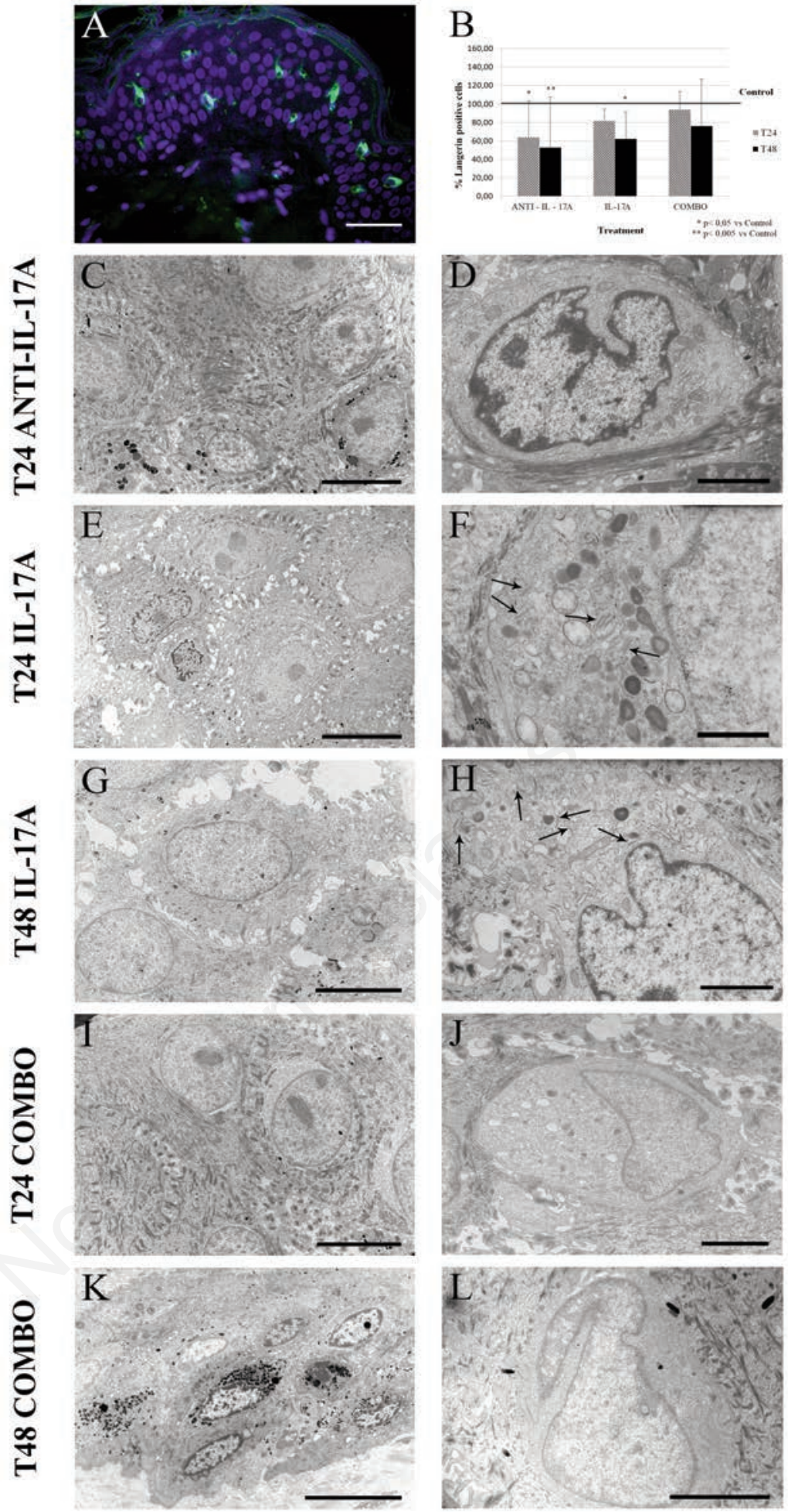

Figure 6. Epidermal LCs quantitative analysis and ultrastructural analysis. Morphological and quantitative analysis of LCs in samples of anti-IL-17A, IL-17A or COMBO groups. A) Representative photomicrograph of a normal human skin paraffin section after anti-humanlangerin immunostaining. B) Quantitative analysis of epidermal LCs; results were expressed as percentage of LCs/ $\mathrm{mm}^{2}$ of living epidermis + 1 standard deviation considering untreated control samples as $\mathbf{1 0 0 \%}$ (thick black line); single asterisk indicates a statistically significant difference $(\mathrm{P}<0.05)$ vs T24 group; double asterisk indicates a statistically significant difference $(\mathrm{P}<0.005)$ vs T24 group (One-way ANOVA test, Dunnett's post-test). C-L) Representative photomicrographs of normal human skin araldite ultrathin sections. C,D) anti-IL-17A-treated samples harvested after 24 (T24) h of culture following the overnight incubation. E,F) IL-17A-treated samples harvested after 24 (T24) $h$ of culture following the overnight incubation. G,H) IL-17A-treated samples harvested after 48 (T48)h of culture following the overnight incubation; K,L) COMBO samples harvested after 48 (T48) h of culture following the overnight incubation. I,J) COMBO samples harvested after 48 (T48) h of culture following the overnight incubation; black arrows indicate Birbeck granules; dotted white line in $\mathrm{A}$ indicates the basal membrane. Scale bars: A) $20 \mu \mathrm{m}$; C,D,J,L) $2 \mu \mathrm{m}$; E,F,G) $5 \mu \mathrm{m}$; H) $1 \mu \mathrm{m}$; K,I) $10 \mu \mathrm{m}$. 
A clear nuclear localization occurred at T48 in the uppermost epidermal layers of IL-17A group (Figure 8E, see inset and white arrows) and in the lower spinous layer of the COMBO group (Figure $8 \mathrm{~F}$, see inset and white arrows), with the exception of the IL-17A and COMBO groups at T48.

\section{Discussion}

In the present study we demonstrate that a specific biological anti-IL-17A agent, with a well-documented clinical effectiveness, promptly exerts an anti-psoriatic switch, after the stimulation with the key psoriatic cytokine IL-17A, in the three-dimensional (3D) organotypic model of skin culture standardized in our lab.,5,11-14 Up to now, most of the data concerning psoriasis and evidences on therapeutical biological efficacy are obtained during the late phases of psoriasis, leaving the cellular mechanism of the very early phase of the disease poorly investigated.

A broad and prompt reversal effect of the epidermal changes induced by the IL-17A agent is the disappearance of the intercellular widening induced by IL-17A in our experimental setting. This observation is parallel to a fine tuning of the expression of the psoriatic cytokines K16 and K17. The unaffected K14 and K10 distribution patterns are in agreement with the lack of blistering and hyperkeratosis, respectively, in all samples at all time points.

The modulation of the mechanical scaffolding role of cytoplasmic intermediate filaments denotes the specific response for the changing needs of the epidermis coping with a psoriatic stimulus as IL-17A. We still lack of a conclusive explanation for the specific K16 distribution in each experimental group, but these results could be interpreted on the basis of the quantitative analysis of cell proliferation. In IL-17A samples, where the most relevant inhibition of epidermal proliferation occurred at both T24 and at T48, the basal layer always showed a very faint K16 expression. When the epidermal proliferation partially recovered, as observed after the incubation with the combination of IL-17A and its specific biological agent, the fastest cytoskeletal rearrangement occurs as early as T24. This proliferative rescue can be interpreted favourably as a

\section{T24}
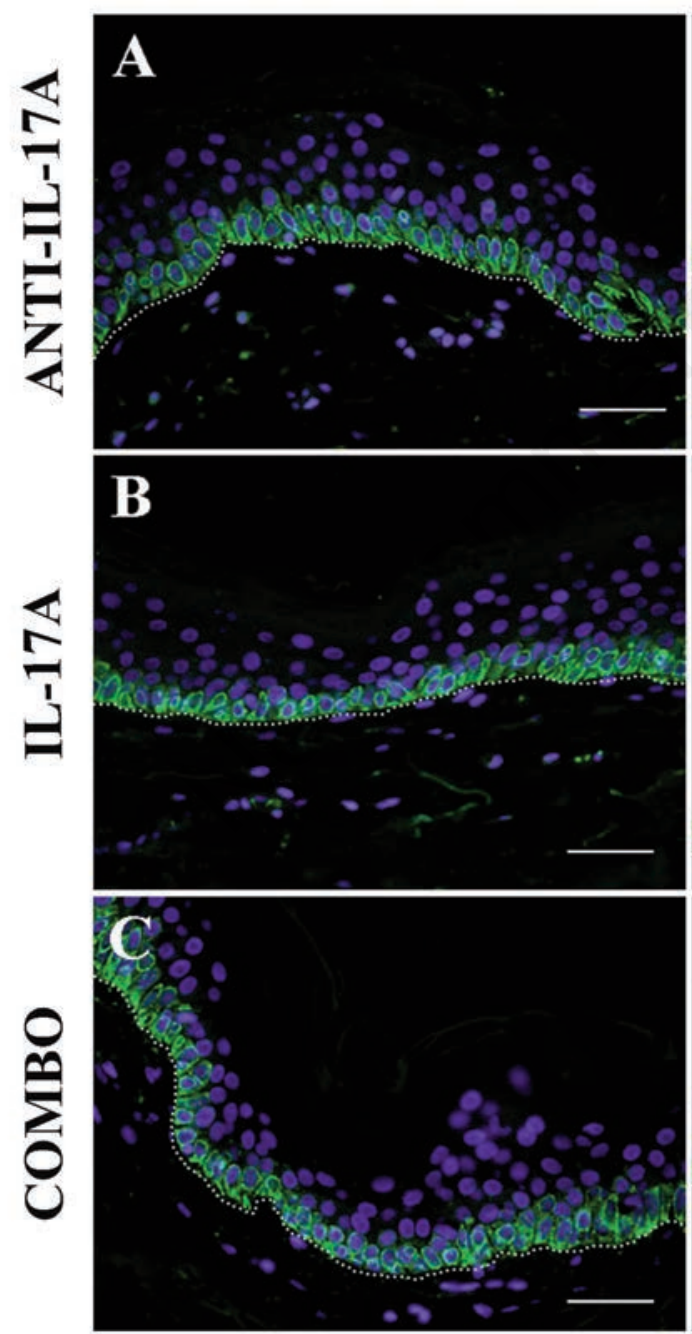

T48
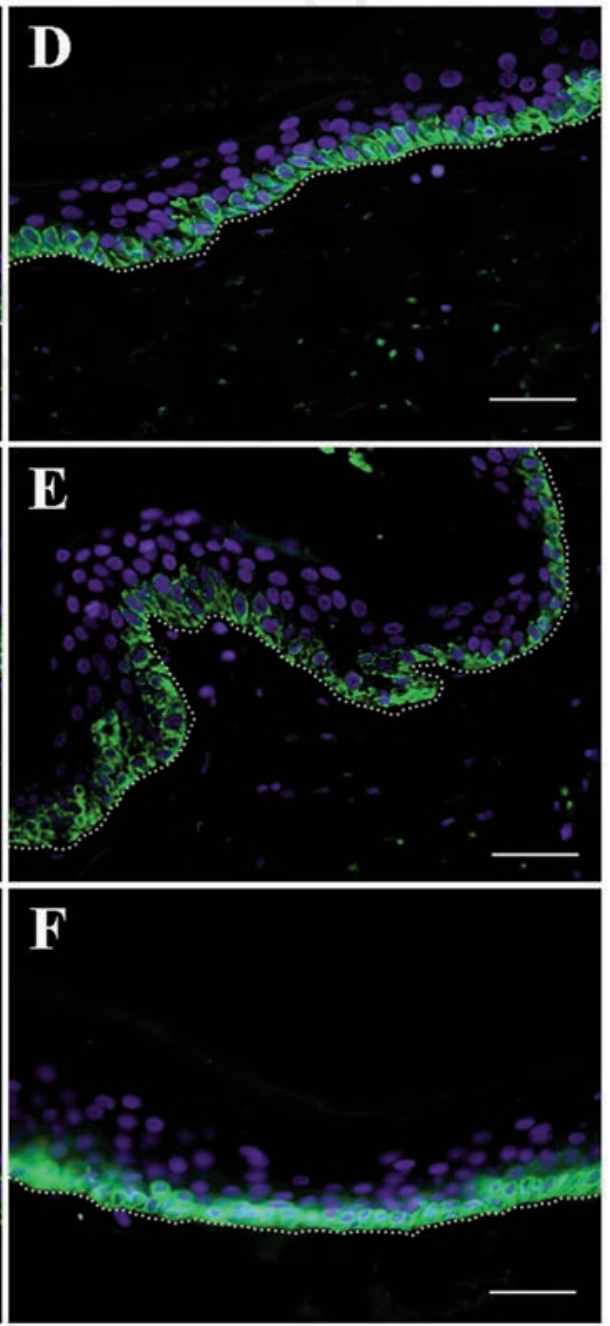

Figure 7. TLR 4 immunofluorescence analysis. Representative photomicrographs of normal human skin paraffin sections after TLR4 immunofluorescence. A,D) IL-17A inhibitor-treated-samples harvested respectively after 24 (T24) and 48 (T48) h of culture. B,E) IL-17Atreated-samples harvested respectively after 24 (T24) and 48 (T48) h of culture. C,F) COMBO samples harvested respectively after 24 (T24) and 48 (T48) h of culture. Nuclei are counterstained with DAPI. Dotted white line indicates the basal membrane. Scale bars: $50 \mu \mathrm{m}$. 
restraint of the "injury" provoked by IL-17A, as previously observed in our model. ${ }^{4}$ This response possibly attenuates the late epidermal "response to injury", and, finally, the psoriatic lesion initiation and progression. Last, but not least, the anti-IL-17A agent alone is able to induce a partial inhibition of $\mathrm{KCs}$ ' proliferation at T24, and, even more, at T48, thus possessing the highest proliferative potential and suggesting that this early occurrence has to become stable at longer time points, as observed in clinics. ${ }^{1,2,15}$

The expression of occludin and filaggrin, i.e. the proteins constituting the physical barrier of the epidermis, was altered in IL17A group. Interestingly, the epidermal barrier was sensitive to the anti-IL-17A agent as soon as $24 \mathrm{~h}$. Conversely, TLR4 seems not to be involved in this early epidermal response, thus suggesting a constitutive expression. As a consequence, the reported NFkB nuclear translocation, indicating its activation, should be independent by TLR4. Regarding the epidermal innate immunity mediated by epidermal LCs, the quantitative LCs analysis clearly shows that the LCs number in living epidermis decreases after the exposure to the specific anti-IL-17A agent alone if compared to controls, thus supporting a positive effect on the innate skin immunity, as this agent does not possess chemoattractant activity. Moreover, in the COMBO group, however, this number was not statistically higher than in IL-17A and anti-IL-17A group. A possible explanation for this phenomenon arises directly from the ultrastructural analysis. The LCs' activation signs were strongly different between the two groups, i.e. IL-17A and COMBO. Indeed, LC functional activation is a prerequisite for their following migration from the epidermal compartment. Considering that i) IL-17A induces an activation of LCs as shown by numerous Birbeck granules in the perinuclear area and a condensed chromatin; ${ }^{11}$ and that ii) no clear signs of activation were observed after the incubation with the anti-IL-17A agent alone, the resulting effect in the COMBO samples is a trapping of LCs in the epidermis. This can be consequent by one side from the chemoattractant activity attributable to IL-17A and, on the other side, from the lack of LC activation by the anti-IL-17A agent.
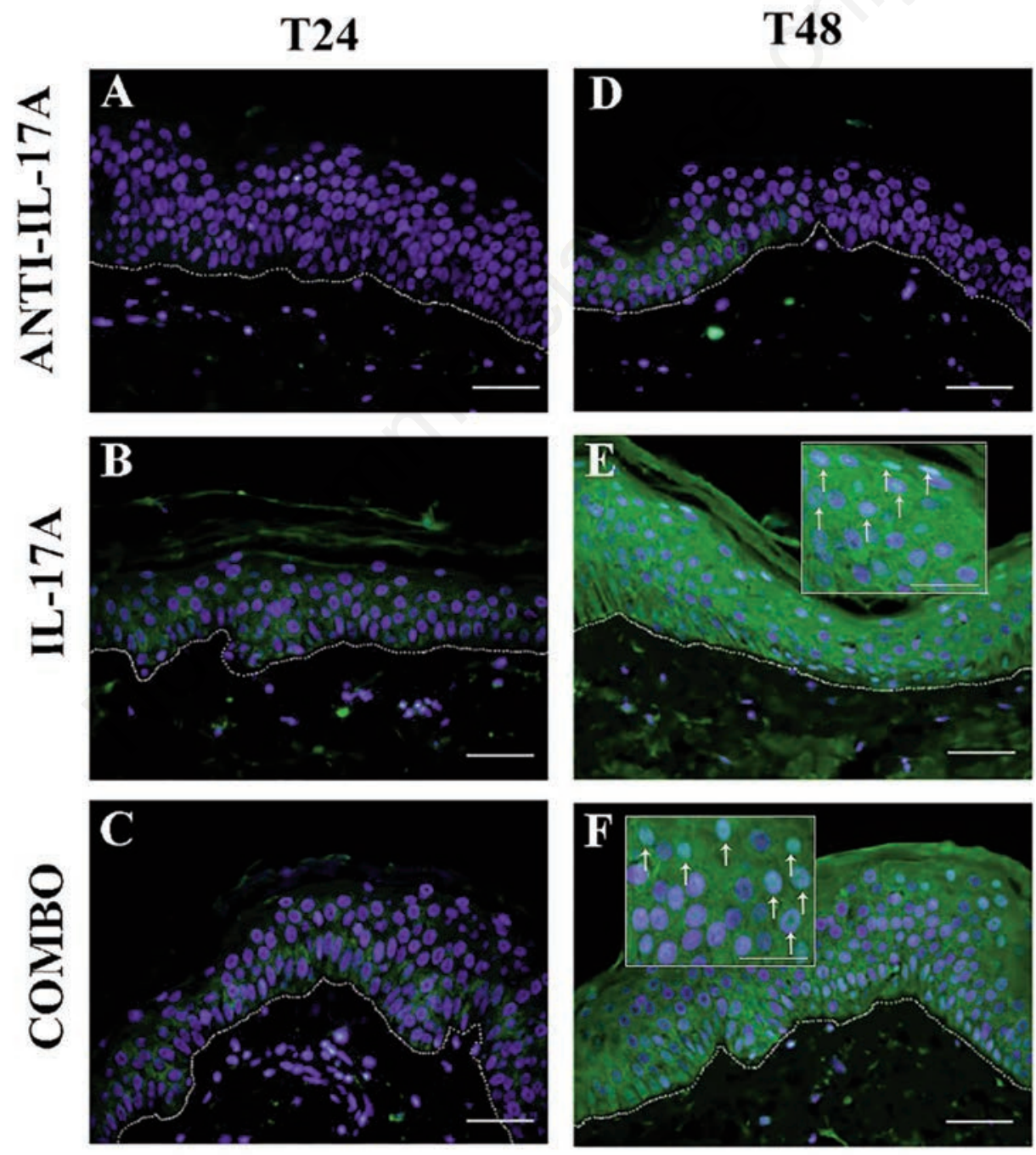

Figure 8. NFkB immunofluorescence analysis. Representative photomicrographs of normal human skin paraffin sections after NFkB immunofluorescence. A,D) IL-17A inhibitor-treated-samples harvested respectively after 24 (T24) and 48 (T48) h of culture. B,E) IL-17Atreated-samples harvested respectively after 24 (T24) and 48 (T48) h of culture. C,F) COMBO samples harvested respectively after 24 (T24) and 48 (T48) h of culture. Nuclei are counterstained with DAPI. Dotted white line indicates the basal membrane. White arrows in E and $F$ indicates the nuclear translocation of NFkB. Scale bars: $50 \mu \mathrm{m}$. 
As a consequence, LCs are not enabled to leave the epidermis, resulting more abundant in the $\mathrm{COMBO}$ group and persisting also at T48. Our study describes, for the first time, the most specific psoriatic cellular events that can be affected or completely reverted by a specific anti-IL-17A agent, during the very early phases of the plaques formation and progression within the epidermal compartment. As most of the studies have been carried when psoriasis is a clinical consolidated skin disease, our experimental approach is helpful in investigating the very early phase of psoriasis, giving strong insights to the complex cellular mechanisms between epidermal cells and the soluble factors typical of the psoriatic milieu.

\section{Acknowledgments}

Part of this work was carried out at NOLIMITS, an advanced imaging facility established by the University of Milan, Italy. We would thank Dr. Vincenzo Conte for his technical help in electron microscopy work.

\section{References}

1. Lynde CW, Poulin Y, Vender R, Bourcier M, Khalil S. Interleukin 17A: Toward a new understanding of psoriasis pathogenesis. J Am Acad Dermatol 2014;71:141-50.

2. Wasilewska A, Winiarska M, Olszewska M, Rudnicka L. Interleukin-17 inhibitors. A new era in treatment of psoriasis and other skin diseases. Postepy Dermatol Alergol 2016;33: 247-52.

3. Thomas LW, Lee EB, Wu JJ. Systematic review of anti-drug antibodies of IL-17 inhibitors for psoriasis. J Dermatolog Treat 2019;30:110-6.

4. Donetti E, Cornaghi L, Gualerzi A, Baruffaldi Preis FW, Prignano F. An innovative three-dimensional model of normal human skin to study the proinflammatory psoriatic effects of tumor necrosis factor-alpha and interleukin-17. Cytokine 2014; 68:1-8.
5. Donetti E, Cornaghi L, Arnaboldi F, Landoni F, Romagnoli P, Mastroianni $\mathrm{N}$, et al. Interleukin 22 early affects keratinocyte differentiation, but not proliferation, in a three-dimensional model of normal human skin. Exp Cell Res 2016;345:247-54.

6. Coulombe PA, Wong P. Cytoplasmic intermediate filaments revealed as dynamic and multipurpose scaffolds. Nat Cell Biol 2004;6:699-706.

7. Karantza V. Keratins in health and cancer: More than mere epithelial cell markers. Oncogene 2011;30:127-38.

8. Paladini RD, Takahashi K, Bravo NS, Coulombe PA. Onset of re-epithelialization after skin injury correlates with a reorganization of keratin filaments in wound edge keratinocytes: Defining a potential role for keratin 16. J Cell Biol 1996;132: 381-97.

9. Donetti E, Lombardo G, Baruffaldi Preis F, Cornaghi L, Pescitelli L, Prignano F. 3D skin model to investigate the early epidermal morphological psoriatic features. J Transl Sci 2019;6:1-4.

10. Vidya MK, Kumar VG, Sejian V, Bagath M, Krishnan G, Bhatta R. Toll-like receptors: Significance, ligands, signaling pathways, and functions in mammals. Int Rev Immunol 2018; 37:20-36.

11. Prignano F, Arnaboldi F, Cornaghi L, Landoni F, Tripo L, Preis FWB, et al. Tumor necrosis factor-alpha and interleukin-17 differently affects Langerhans cell distribution and activation in an innovative three-dimensional model of normal human skin. Eur J Cell Biol 2015;94:71-7.

12. Bedoni M, Sforza C, Dolci C, Donetti E. Proliferation and differentiation biomarkers in normal human breast skin organotypic cultures. J Dermatol Sci 2007;46:139-42.

13. Donetti E, Cornaghi L, Arnaboldi F, Ricceri F, Pescitelli L, Maiocchi M, Carriero F, Baruffaldi Preis F, Prignano F. Epidermal barrier reaction to an in vitro psoriatic microenvironment. Exp Cell Res 2017; 360:180-8

14. Thomas LW, Lee EB, Wu JJ. Systematic review of anti-drug antibodies of IL-17 inhibitors for psoriasis. J Dermatolog Treat 2019;30:110-6.

Received for publication: 3 February 2020. Accepted for publication: 6 April 2020.

This work is licensed under a Creative Commons Attribution-NonCommercial 4.0 International License (CC BY-NC 4.0).

CCopyright: the Author(s), 2020

Licensee PAGEPress, Italy

European Journal of Histochemistry 2020; 64:3115

doi:10.4081/ejh.2020.3115 